\title{
Long-time Behavior of Isolated Periodically Driven Interacting Lattice Systems
}

\author{
Luca D’Alessio $^{1,2, *}$ and Marcos Rigol ${ }^{1}$ \\ ${ }^{1}$ Department of Physics, The Pennsylvania State University, University Park, Pennsylvania 16802, USA \\ ${ }^{2}$ Department of Physics, Boston University, Boston, Massachusetts 02215, USA
}

(Received 19 February 2014; revised manuscript received 15 August 2014; published 19 December 2014)

\begin{abstract}
We study the dynamics of isolated interacting spin chains that are periodically driven by sudden quenches. Using full exact diagonalization of finite chains, we show that these systems exhibit three distinct regimes. For short driving periods, the Floquet Hamiltonian is well approximated by the time-averaged Hamiltonian, while for long periods, the evolution operator exhibits properties of random matrices of a circular ensemble (CE). In between, there is a crossover regime. Based on a finite-size scaling analysis and analytic arguments, we argue that, for thermodynamically large systems and nonvanishing driving periods, the evolution operator always exhibits properties of the $\mathrm{CE}$ of random matrices. Consequently, the Floquet Hamiltonian is a nonlocal Hamiltonian with multispin interaction terms, and the driving leads to the equivalent of an infinite temperature state at long times. These results are connected to the breakdown of the Magnus expansion and are expected to hold beyond the specific lattice model considered.
\end{abstract}

DOI: 10.1103/PhysRevX.4.041048

\section{INTRODUCTION}

Periodically driven systems have a long history. One paradigmatic example is the kicked-rotor model of a particle moving on a ring subjected to time-periodic "kicks" [1]. The behavior of such systems is very rich; e.g., they can display interesting integrability-to-chaos transitions and dynamical Anderson localization [2-4], and counterintuitive effects such as dynamical stabilization [5,6], both in classical and quantum mechanics. Moreover, it has recently been shown that periodic perturbations can be used as a flexible experimental knob [7-9] to realize synthetic matter, i.e., matter with specific engineered properties. Two outstanding examples in this direction are the opening of a gap in graphene by using light or carefully selected lattice phonons [10-12] and the realization of the so-called Floquet topological insulator in a material that, in the absence of driving, is topologically trivial $[13,14]$.

With a few exceptions [15-26], recent studies mostly focus on driving simple (often single-particle) Hamiltonians. In some instances, it is known that the addition of interactions qualitatively changes the physics; e.g., when two quantum kicked rotors are coupled, the dynamical Anderson localization is suppressed and the (classical) diffusive behavior is recovered $[27,28]$. Furthermore, recent studies often rely on approximations that allow one to recast the effect of the drive in, e.g., an effective hopping [29,30]. While these

\footnotetext{
*dalessio@bu.edu
}

Published by the American Physical Society under the terms of the Creative Commons Attribution 3.0 License. Further distribution of this work must maintain attribution to the author(s) and the published article's title, journal citation, and DOI.
Subject Areas: Quantum Physics

approximations can work well for noninteracting systems, their range of applicability for interacting quantum systems is unclear. The validity of some of those approximations, such as the use of average Hamiltonians, has been a topic of interest because of its relevance to NMR experiments [31,32].

The goal in this paper is to understand the long-time behavior of isolated interacting quantum systems periodically driven by sudden quenches. We focus on driving finite spin chains, for which the Hilbert space is finite. By considering chains of different lengths, we systematically study the role of finite-size effects. Combining numerical results and analytic arguments, we argue that finite chains exhibit three distinct regimes, while in thermodynamically large systems, only one regime survives and the system approaches the equivalent of an infinite temperature state at long times. In this regime, the Floquet Hamiltonian is a nonlocal Hamiltonian that contains multispin interaction terms. It is therefore qualitatively different from physically realizable static Hamiltonians, which generally contain only few-spin interaction terms. We argue that this regime is generic to interacting quantum systems. Thus, our study provides a paradigm to understand the long-time behavior of isolated, periodically driven, interacting quantum systems.

The exposition is organized as follows. In Sec. II, we review the Floquet theorem and present general analytic arguments of relevance to our study. The numerical results for the specific model considered are presented in Sec. III. Section IV is devoted to a discussion of far-reaching implications of our study. A summary of our results is presented in Sec. V.

\section{THEORETICAL ANALYSIS}

The Floquet theorem states that the evolution operator of any periodically driven system can be written as 


$$
\hat{U}(t) \equiv \hat{P}(t) e^{-i \hat{H}_{F} t / \hbar},
$$

where $\hat{P}(t+T)=\hat{P}(t)$ is a periodic unitary operator that reduces to the identity at stroboscopic times $\left(t_{N}=N T\right.$, with $N$ an integer), and $\hat{H}_{F}$ is the time-independent Floquet Hamiltonian. It is clear that the factorization of the evolution operator is not unique and, therefore, there is some freedom in the definition of both the periodic operator $\hat{P}(t)$ and the Floquet Hamiltonian $\hat{H}_{F}[33,34]$. At stroboscopic times, Eq. (1) simplifies as the periodic operator drops out. In particular, the exact evolution operator over a single driving cycle can be written as

$$
\hat{U}_{\text {cycle }}=e^{-i \hat{H}_{F} T / \hbar}=\sum_{n}\left|\phi_{n}\right\rangle e^{-i \theta_{n}}\left\langle\phi_{n}\right|,
$$

where $\left|\phi_{n}\right\rangle$ and $e^{-i \theta_{n}}$ are the eigenstates and eigenvalues of $\hat{U}_{\text {cycle. }}$. From Eq. (2), it follows that

$$
\hat{H}_{F} \equiv \sum_{n}\left|\phi_{n}\right\rangle \varepsilon_{n}\left\langle\phi_{n}\right|
$$

where $\varepsilon_{n}$ are the Floquet quasienergies. The nonuniqueness of the factorization in Eq. (1) translates into the fact that the Floquet quasienergy $\varepsilon_{n}$ can be shifted by integers of $\hbar \omega$, where $\omega=2 \pi / T$. We note, however, that this freedom does not affect the eigenstates $\left|\phi_{n}\right\rangle$ or the eigenvalues $e^{-i \theta_{n}}=$ $e^{-i \varepsilon_{n} T / \hbar}$ of $\hat{U}_{\text {cycle }}$, which are the focus of our study.

Equation (2) resembles the standard unitary evolution operator of a system described by a time-independent Hamiltonian $\hat{H}_{F}$. However, this simplicity is deceptive. For interacting systems, it is, in general, impossible to obtain the Floquet Hamiltonian in a closed form, and one has to rely on approximations. A commonly used approximation scheme is the Magnus expansion [34-36], which is a series expansion in the driving period $T$. It allows one to compute the Floquet Hamiltonian as $\hat{H}_{F}=\sum_{n=0}^{\infty} \hat{H}_{F}^{(n)}$. The first two terms in the Magnus expansion are

$$
\begin{aligned}
& H_{F}^{(0)}=\frac{1}{T} \int_{0}^{T} \mathrm{~d} t \hat{H}(t) \\
& H_{F}^{(1)}=\frac{1}{2 ! T i \hbar} \int_{0}^{T} \mathrm{~d} t_{1} \int_{0}^{t_{1}} \mathrm{~d} t_{2}\left[\hat{H}\left(t_{1}\right), \hat{H}\left(t_{2}\right)\right],
\end{aligned}
$$

where $\hat{H}(t+T)=\hat{H}(t)$ is the time-periodic Hamiltonian of the system. The zeroth-order term $\hat{H}_{F}^{(0)}$ is simply the time-averaged Hamiltonian, which we denote as $\hat{H}_{\text {ave }}$ in what follows. Higher-order terms contain nested commutators of $\hat{H}(t)$ and multiple time-ordered integrals. The Magnus expansion is guaranteed to converge to the exact Floquet Hamiltonian for systems with a bounded energy spectrum (such as the spin chain we consider here) and sufficiently short driving periods, i.e., $T \leq T_{c}=2 \pi / W$, where $W$ is the bandwidth of $\hat{H}(t)$ (see Ref. [36] and references therein). For interacting systems, the energy bandwidth $W$ is extensive, and therefore, the Magnus expansion is guaranteed to converge only for $T \leq T_{c}=$ const/ $V$, where $V$ is the volume of the system. We stress that this condition is only a sufficient one. It is unknown whether, for interacting systems in the thermodynamic limit, the radius of convergence of the Magnus expansion is finite. Our results suggest that this is, in general, not the case and that the Magnus expansion in thermodynamically large systems has a vanishing radius of convergence.

The Magnus expansion has several interesting properties [35,36]. First, being an expansion for the Floquet Hamiltonian, it ensures that the evolution operator is unitary at any order in the expansion. This should be contrasted with the well-known Dyson series [37], which generates nonunitary evolution operators when truncated to any finite order. Second, noticing that the commutator of two local extensive operators is local and extensive, we see that the Floquet Hamiltonian is local and extensive in each order of the Magnus expansion. Therefore, when the Magnus expansion converges, the Floquet Hamiltonian is a local extensive Hamiltonian with few-body interactions. Hence, it exhibits the usual properties of experimentally realizable time-independent Hamiltonians [20]. However, when the Magnus expansion does not converge, the Floquet Hamiltonian can be qualitatively different from experimentally relevant static Hamiltonians; i.e., it can be "unphysical" with nonlocal multibody interactions.

To study properties of the Floquet Hamiltonian in regimes where the Magnus expansion might not converge, we follow the approach presented in Ref. [31]. We introduce a generic bounded time-periodic local Hamiltonian $\hat{H}(\tau)$ defined on a finite-dimensional Hilbert space

$$
\hat{H}(\tau)=\hat{H}_{\mathrm{ave}}+f(\tau) \hat{A},
$$

where $\tau \equiv t / T$ is the rescaled time, $f(\tau)$ is an arbitrary time-periodic function with zero average, $\hat{H}_{\text {ave }}$ is the timeaveraged Hamiltonian, and $\hat{A}$ is a sum of local few-body operators. $|n(\tau)\rangle$ and $\epsilon_{n}(\tau)$ are the instantaneous eigenfunctions and eigenenergies of $\hat{H}(\tau)$ :

$$
\hat{H}(\tau)|n(\tau)\rangle=\epsilon_{n}(\tau)|n(\tau)\rangle .
$$

The Schrödinger equation reads

$$
\partial_{\tau} \hat{U}(\tau)=T \frac{\hat{H}(\tau)}{i \hbar} \hat{U}(\tau)
$$

and is formally solved by

$$
\hat{U}(\tau)=\mathcal{T} \exp \left[T \int_{0}^{\tau} d \tau^{\prime} \frac{\hat{H}\left(\tau^{\prime}\right)}{i \hbar}\right] \equiv \exp \left[T \frac{\hat{H}_{\text {eff }}(\tau)}{i \hbar}\right],
$$

where $\mathcal{T}$ ensures that the exponential is time ordered. The last equality defines an effective Hamiltonian $\hat{H}_{\text {eff }}(\tau)$. 
The instantaneous eigenstates and eigenvalues of the evolution operator $\hat{U}(\tau)$ are

$$
\hat{U}(\tau)\left|\phi_{n}(\tau)\right\rangle=e^{-i \theta_{n}(\tau)}\left|\phi_{n}(\tau)\right\rangle .
$$

From Eqs. (2) and (7), one can see that $\hat{U}(\tau=1)=\hat{U}_{\text {cycle }}$ and, correspondingly, $\hat{H}_{\text {eff }}(\tau=1) \equiv \hat{H}_{F}$. This justifies using the same notation for the eigenvectors and eigenvalues appearing in Eqs. (2) and (8). Substituting Eq. (7) into the Schrödinger equation (6) leads to [31] (see also Appendix A)

$$
\begin{aligned}
& \frac{i \hbar}{T}\left(1-e^{-i\left(\theta_{n}(\tau)-\theta_{m}(\tau)\right)}\right)\left\langle\phi_{n}(\tau)\left|\partial_{\tau}\right| \phi_{m}(\tau)\right\rangle \\
& \quad=\left\langle\phi_{n}(\tau)|\hat{H}(\tau)| \phi_{m}(\tau)\right\rangle .
\end{aligned}
$$

We note that this equation is exact since it corresponds to a rewriting of the Schrödinger equation.

Remarkably, evaluating Eq. (9) at $\tau=1$ allows one to connect the structure of the eigenstates of $\hat{U}_{\text {cycle }}$ (and of $\hat{H}_{F}$ ) to the statistics of the folded phases, i.e., the phases $\theta_{n}$ defined in $[-\pi, \pi)$. This connection can be made by analyzing under which circumstances the left-hand side and right-hand side (RHS) are not identically zero. For example, when the eigenstates of $\hat{H}_{F}$ exhibit eigenstate thermalization [38-44], the RHS of Eq. (9) is generically nonzero. This occurs because $\hat{H}(\tau=1)$ is taken to be a sum of local few-body operators [38-42]. Therefore, the spectrum of $\hat{U}_{\text {cycle }}$ is expected to display repulsion, i.e., $e^{-i\left(\theta_{n}-\theta_{m}\right)} \neq 1$ or equivalently $\theta_{n} \neq \theta_{m}$. On the other hand, if $\hat{H}_{F}$ is noninteracting (or integrable), then the RHS of Eq. (9) can be zero for a large fraction of pairs of states $[41,42]$ and there does not need to be repulsion. In this case, the spectrum of $\hat{U}_{\text {cycle }}$ can exhibit highly degenerate phases separated by finite gaps.

We should stress that, for $T>T_{c}=2 \pi / W$, repulsion in the spectrum of $\hat{U}_{\text {cycle }}$ is incompatible with $\hat{H}_{F}=\hat{H}_{\text {ave }}$. This does not follow from Eq. (9). It becomes apparent because, for $\hat{H}_{F}=\hat{H}_{\text {ave }}, \theta_{n}=\theta_{n}^{\text {ave }}$, where $\theta_{n}^{\text {ave }}$ is obtained by folding $T \epsilon_{n}^{\text {ave }} / \hbar$ in $[-\pi, \pi)$, and $\epsilon_{n}^{\text {ave }}$ are the eigenvalues of $\hat{H}_{\text {ave }}$. Since energies $\epsilon_{n}^{\text {ave }}$ that are far apart are not expected to be correlated with each other, the (folded) phases $\theta_{n}$ will not exhibit repulsion. The same reasoning leads to the conclusion that, for $T>T_{c}$, phase repulsion is incompatible with $\hat{H}_{F}$ being an extensive operator with few-body interactions. Actually, repulsion in the phases of $\hat{U}_{\text {cycle }}$ for $T>T_{c}$ hints that $\hat{U}_{\text {cycle }}$ should exhibit properties of random matrices belonging to circular ensembles (CEs) [45-50]. CEs are the equivalent of Gaussian ensembles (GEs) for unitary matrices. In CEs, the phases display repulsion and the eigenstates are essentially random vectors.

Two remarks are in order. First, while it is believed that eigenstate thermalization and chaos (or level repulsion in the spectrum) come together in many-body interacting systems $[38-40,43,44]$, there is no proof that this is always the case. Our analysis based on Eq. (9) does not constitute a proof that this is the case for $\hat{H}_{F}$. However, it is a step in the right direction. As long as $\hat{H}(\tau=1)$ is a sum of local operators and $T$ is small, Eq. (9) allows us to make a connection between eigenstate thermalization and the presence of level repulsion in the spectrum. Second, phase repulsion in the Floquet spectrum is not unique to interacting systems. It can appear in chaotic single-particle driven systems (see, for example, Ref. [49]) for specific parameters of the driving protocol [4]. For example, in kicked systems, the dynamics of the Floquet eigenvalues for varying kicked strength can be mapped to the dynamics of interacting quasiparticles, i.e., the "Pechukas gas," and the phase repulsion in the Floquet spectrum can be inferred from the equilibrium properties of this fictitious gas [49]. What we expect to be different in driven interacting quantum systems is that phase repulsion will be robust to changes in the driving protocol.

We should also stress that the presence of level repulsion in the spectrum of $\hat{U}_{\text {cycle }}$, for nonvanishing values of $T$ in thermodynamically large systems, is an unbiased indicator of the breakdown of the Magnus expansion. This is because, as explained before, when the Magnus expansion converges, $\hat{H}_{F}$ is a local extensive Hamiltonian with fewbody interactions. This is incompatible with having phase repulsion for driving periods $T>T_{c}=\mathrm{const} / V$, and $T_{c}$ vanishes with increasing system size.

\section{NUMERICAL RESULTS}

We now turn to numerical simulations of a realistic many-body system. Specifically, we use full exact diagonalization to study a spin- $1 / 2$ chain with periodic boundary conditions and the Hamiltonian

$$
\begin{aligned}
\hat{H}(t) & =[J+f(t) \delta J] \hat{H}_{n n}+J^{\prime} \sum_{j} \sigma_{j}^{z} \sigma_{j+2}^{z}, \\
\hat{H}_{n n} & =\sum_{j}\left[\sigma_{j}^{z} \sigma_{j+1}^{z}-\frac{1}{2}\left(\sigma_{j}^{x} \sigma_{j+1}^{x}+\sigma_{j}^{y} \sigma_{j+1}^{y}\right)\right],
\end{aligned}
$$

where $\sigma_{j}^{\alpha}, \alpha=x, y, z$, is the Pauli matrix for the $j$ th spin, and $f(t)$ is a periodic function with zero average,

$$
f(t) \equiv \begin{cases}1 & \text { for } N<\frac{t}{T}<N+\frac{1}{2} \\ -1 & \text { for } N+\frac{1}{2}<\frac{t}{T}<N+1 .\end{cases}
$$

The Hamiltonian $\hat{H}_{n n}$ in Eq. (10) is relevant to nuclear magnetic resonance experiments in solids [51]. The nextnearest neighbor coupling $J^{\prime}$ can be considered to be a phenomenological parameter that accounts for interactions that break the integrability of the spin- $1 / 2 X X Z$ chain. We restrict our calculations to the sector with total quasimomentum $k=0$, magnetization $m_{z}=1 / 3$, and parity $p=+1$. For the four system sizes considered in our 
study, $L=15,18,21$, and 24 , the number of states in that sector are $D=111,561,2829$, and 15581 , respectively.

The Hamiltonian parameters are selected to be $J=1$, $\delta J=0.2$, and $J^{\prime}=0.8$. They have been chosen to ensure that the average Hamiltonian is nonintegrable and that it exhibits eigenstate thermalization and quantum chaos [38-44]. This is tested in Fig. 1, where we show the probability distribution $P(r)$, with $r$ being the ratio of two consecutive energy gaps [52-54], in the spectrum of $\hat{H}_{\text {ave }}$ :

$$
r=\frac{\min \left(s_{n}, s_{n+1}\right)}{\max \left(s_{n}, s_{n+1}\right)} \in[0,1], \quad s_{n}=\epsilon_{\mathrm{ave}}^{n+1}-\epsilon_{\mathrm{ave}}^{n} .
$$

The distribution $P(r)$ is related to the level spacing distribution $P(s)$ in that they both measure the level repulsion in the spectrum, which is a standard quantum chaos indicator [4]. However, the distribution $P(r)$ is simpler to compute since it does not require the unfolding of the spectrum, which is known to be nontrivial $[55,56]$. In Fig. 1, we compare $P(r)$ to the predictions for the Gaussian orthogonal ensemble (GOE) and Poisson statistics (POI) [54]:

$$
P_{\mathrm{GOE}}(r)=\frac{27}{4} \frac{r+r^{2}}{\left(1+r+r^{2}\right)^{5 / 2}}, \quad P_{\mathrm{POI}}(r)=\frac{2}{(1+r)^{2}} .
$$

We notice that $P(r)$ is well approximated by $P_{\mathrm{GOE}}(r)$ and converges to the GOE prediction with increasing system size. This can be seen in the inset, in which the error

$$
\text { error }=\int_{0}^{1} d r\left|P(r)-P_{\mathrm{GOE}}(r)\right|
$$

is shown to decrease as the system size increases. The presence of level repulsion is clearly seen as $r \rightarrow 0$,

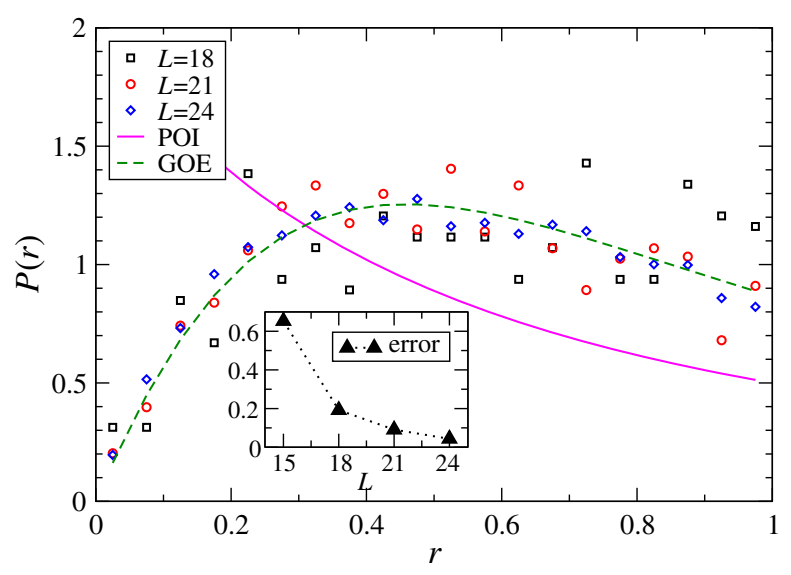

FIG. 1. Probability distribution $P(r)$ for the ratio of two consecutive energy gaps in the spectrum of $\hat{H}_{\text {ave }}$ [see Eq. (12)] together with the GOE and POI predictions. (Inset) The error distance between $P(r)$ and the GOE prediction decreases quickly with an increasing system size. where $P_{\mathrm{GOE}}(r)$ vanishes while $P_{\mathrm{POI}}(r)$ remains finite. The difference between those distributions is reflected in the average value of $r$ :

$$
\langle r\rangle_{\mathrm{GOE}} \approx 0.535898, \quad\langle r\rangle_{\mathrm{POI}} \approx 0.386294 .
$$

As shown in Refs. [52,53], $\langle r\rangle$ encodes information about level repulsion. It has been used as a sensitive and practical probe of the many-body localization transition [57-62].

Given our driving protocol [see Eq. (11)], the exact timeevolution operator over one cycle is

$$
\hat{U}_{\text {cycle }}=e^{-i \frac{T}{2 \hbar} \hat{H}_{+}} e^{-i \frac{T}{2 h} \hat{H}_{-}}=\sum_{n}\left|\phi_{n}\right\rangle e^{-i \theta_{n}}\left\langle\phi_{n}\right|
$$

where $\hat{H}_{ \pm}$corresponds to Eq. (10) for $f(t)= \pm 1$. Using exact diagonalization, we obtain the eigenvectors $\left|\phi_{n}\right\rangle$ and the phases $\theta_{n}$, which we define in the interval $[-\pi, \pi)$.

In what follows, we study how the properties of the time-evolution operator change as a function of the driving period $T$. We use indicators based on the phases (Sec. III A) and on the eigenvectors (Sec. III B) of $\hat{U}_{\text {cycle. These two }}$ indicators suggest that $\hat{U}_{\text {cycle }}$ shares properties with matrices from the circular ensemble for nonvanishingly small values of $T$ in thermodynamically large systems. This causes the system to approach, independent of the initial conditions, the equivalent of an infinite temperature state at long times (Sec. III C).

\section{A. Phase repulsion}

We are first interested in understanding the properties of the phases $\theta_{n}$ as a function of the driving period $T$. We also want to find the relation between those phases and the phases $\theta_{n}^{\text {ave }}$, which would be obtained if $\hat{H}_{F}=\hat{H}_{\text {ave }}$. To this end, we use the equivalent of $P(r)$ in our setup. We define $r$ as the ratio of two consecutive phase gaps:

$$
r=\frac{\min \left(\delta_{n}, \delta_{n+1}\right)}{\max \left(\delta_{n}, \delta_{n+1}\right)} \in[0,1], \quad \delta_{n}=\theta_{n+1}-\theta_{n} .
$$

We stress that "phase gaps" are obtained by first ordering the phases in $[-\pi, \pi)$ and then computing the difference between consecutive values.

In Fig. 2, we show the average value of $r$ vs $T$ for the exact phases $\theta_{n}$ (indicated by $\langle r\rangle$ ) and the phases $\theta_{n}^{\text {ave }}$ (indicated by $\left\langle r_{\text {ave }}\right\rangle$ ). We compare them with the predictions of the COE, the GOE, and POI. The COE and GOE predictions in Fig. 2 have been computed for a finite-size matrix. While the COE and GOE results obtained in those calculations are different, they are expected to coincide in the thermodynamic limit (see Appendix B).

In Fig. 2, note the vertical dotted lines that mark $T_{1}=2 \pi \hbar / W, T_{2}=\pi \hbar / \sigma$, and $T_{3}=2 \pi \hbar / \sigma$, where $W=$ $\epsilon_{\mathrm{ave}}^{\max }-\epsilon_{\mathrm{ave}}^{\min }$ is the bandwidth and $\sigma$ is the variance of the 


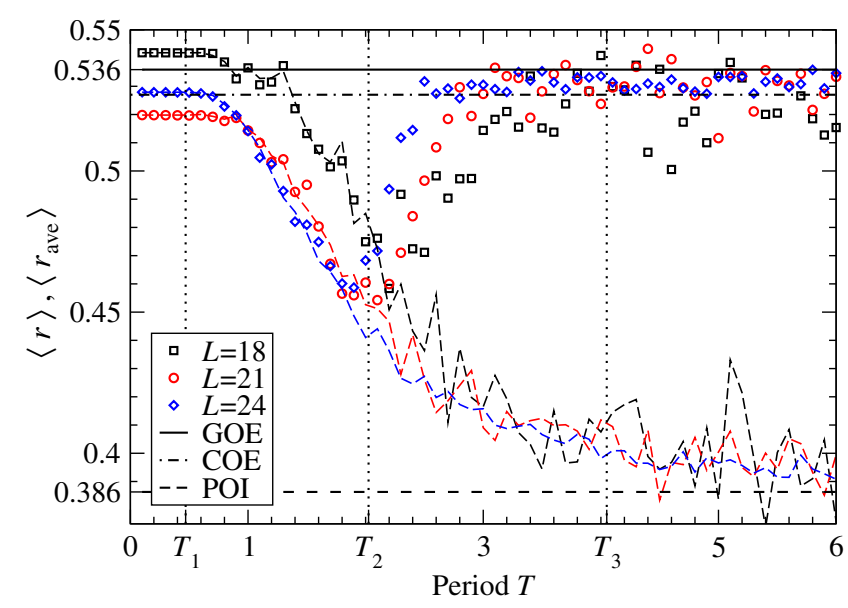

FIG. 2. Average value of $r$ vs $T$. The values of $\langle r\rangle$ (symbols) and $\left\langle r_{\text {ave }}\right\rangle$ (dashed lines) are compared to the COE, GOE, and POI predictions. The COE and GOE predictions are expected to coincide in the thermodynamic limit (see Appendix B). Vertical dotted lines depict the periods $T_{1}=2 \pi \hbar / W, T_{2}=\pi \hbar / \sigma$, and $T_{3}=2 \pi \hbar / \sigma$ (see text).

spectrum of $\hat{H}_{\text {ave }}$ for $L=24$. The variance $\sigma$ is computed as the width of the best Gaussian fit to the density of states of $\hat{H}_{\text {ave }}$. We note that $W \propto L$ and $\sigma \propto \sqrt{L}$ scale differently with the system size $L$. The periods $T_{1}, T_{2}$, and $T_{3}$ separate four regimes in which $\langle r\rangle$ and $\left\langle r_{\text {ave }}\right\rangle$ exhibit distinct behaviors. (i) For $T<T_{1},\langle r\rangle$ is independent of $T$, identical to $\left\langle r_{\text {ave }}\right\rangle$, and close to the GOE prediction. (ii) For $T_{1}<T<T_{2},\langle r\rangle$ and $\left\langle r_{\text {ave }}\right\rangle$ are very close to each other and decrease with increasing $T$. (iii) For $T_{2}<T<T_{3},\langle r\rangle$ increases towards the COE prediction while $\left\langle r_{\text {ave }}\right\rangle$ decreases towards the POI prediction. (iv) For $T>T_{3}$, $\langle r\rangle$ is again independent of $T$ and close to the COE prediction, while $\left\langle r_{\text {ave }}\right\rangle$ is close to and continues approaching the POI prediction.

In Fig. 3, we show the full distribution $P(r)$ for both $\theta_{n}$ and $\theta_{n}^{\text {ave }}$ for the largest system size considered $(L=24)$ for the periods $T_{1}, T_{2}$, and $T_{3}$ (defined above). We also compare them with the GOE, COE, and POI predictions. These plots show that, for $T_{1}$ and $T_{3}$, the numerical results for the exact phases, $\theta_{n}$, are virtually indistinguishable from the GOE and COE predictions, respectively. On the other hand, the phases $\theta_{n}^{\text {ave }}$ are clearly described by Poisson statistics for $T_{3}$. For $T_{2}$, the results for both $\theta_{n}$ and $\theta_{n}^{\text {ave }}$ are in between GOE/COE and POI. Note that $T_{1} \propto L^{-1}$, $T_{2} \propto L^{-1 / 2}$, and $T_{3} \propto L^{-1 / 2}$ all collapse to zero in the thermodynamic limit.

\section{B. Eigenvectors' information entropy}

The properties of the eigenvectors of $\hat{U}_{\text {cycle }}$ provide further evidence of the different regimes seen in Fig. 2. Specifically, we study how the eigenvectors of $\hat{U}_{\text {cycle }}$ differ from those of $\hat{H}_{\text {ave }}$ as $T$ is increased. We write the former in terms of the latter,

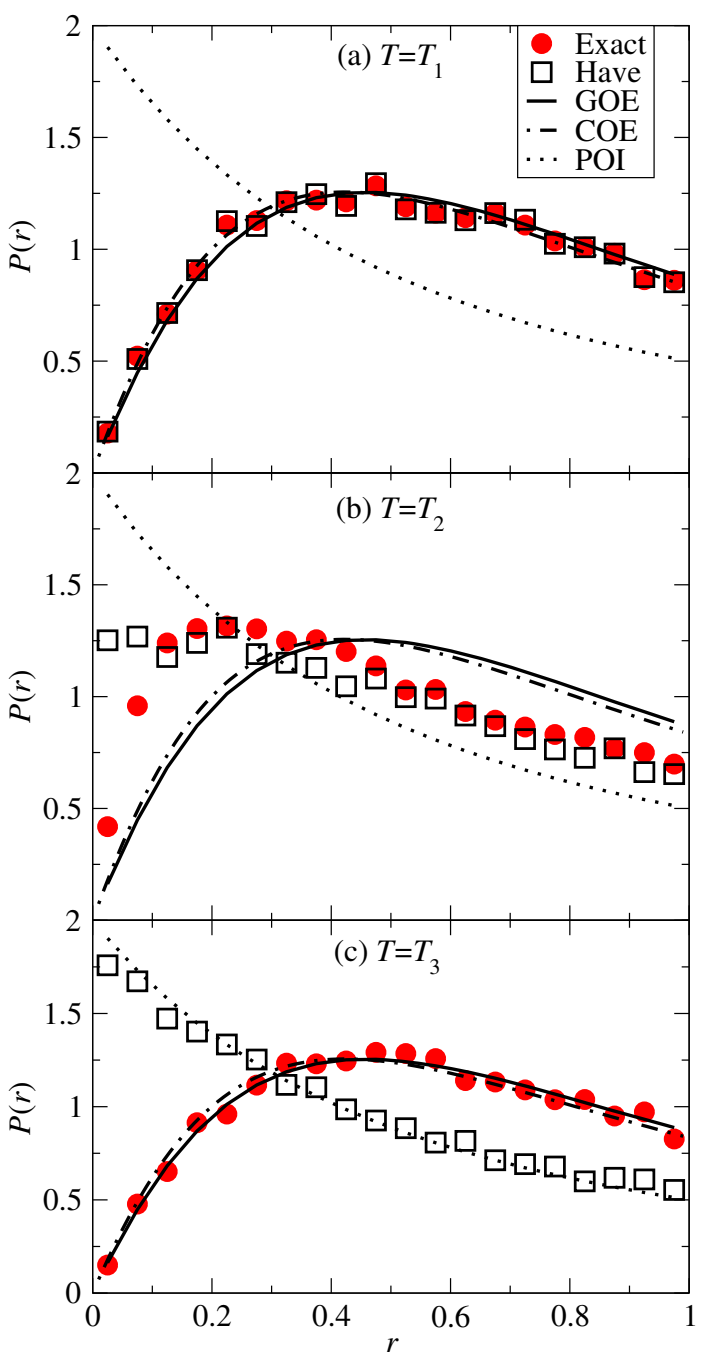

FIG. 3. Distribution $P(r)$ for the periods $T=T_{1}, T_{2}$, and $T_{3}$ shown in Fig. 2. We compare the exact phases obtained from the exact diagonalization of $\hat{U}_{\text {cycle }}$ (indicated by red circles) and the phases one obtains if $\hat{H}_{F}=\hat{H}_{\text {ave }}$ (indicated by squares), with the GOE, COE, and POI predictions (lines).

$$
\left|\phi_{n}\right\rangle=\sum_{m=1}^{D} c_{m}^{n}\left|m_{\mathrm{ave}}\right\rangle,
$$

where $\left|m_{\text {ave }}\right\rangle$ are the eigenstates of $\hat{H}_{\text {ave }}, \hat{H}_{\text {ave }}\left|m_{\text {ave }}\right\rangle=$ $\epsilon_{m}^{\text {ave }}\left|m_{\text {ave }}\right\rangle$, and $D$ is the dimension of the Hilbert space. We then compute the (Shannon) information entropy $[43,44]$

$$
S_{n}=-\sum_{m=1}^{D}\left|c_{m}^{n}\right|^{2} \ln \left|c_{m}^{n}\right|^{2},
$$

which measures the number of states $\left|m_{\text {ave }}\right\rangle$ that contribute to each $\left|\phi_{n}\right\rangle$. When $\hat{H}_{F}=\hat{H}_{\text {ave }}, S_{n}=0$. As $\hat{H}_{F}$ deviates from $\hat{H}_{\text {ave }}, S_{n}$ grows and is expected to saturate to the COE prediction $S_{n} \approx \ln (0.48 D)$, provided $\hat{U}_{\text {cycle }}$ exhibits properties of matrices from the circular ensemble.

In Fig. 4, we show the average entropy $\langle S\rangle=$ $\left(\sum_{n=1}^{D} S_{n}\right) / D$ normalized by the COE prediction. Note that 


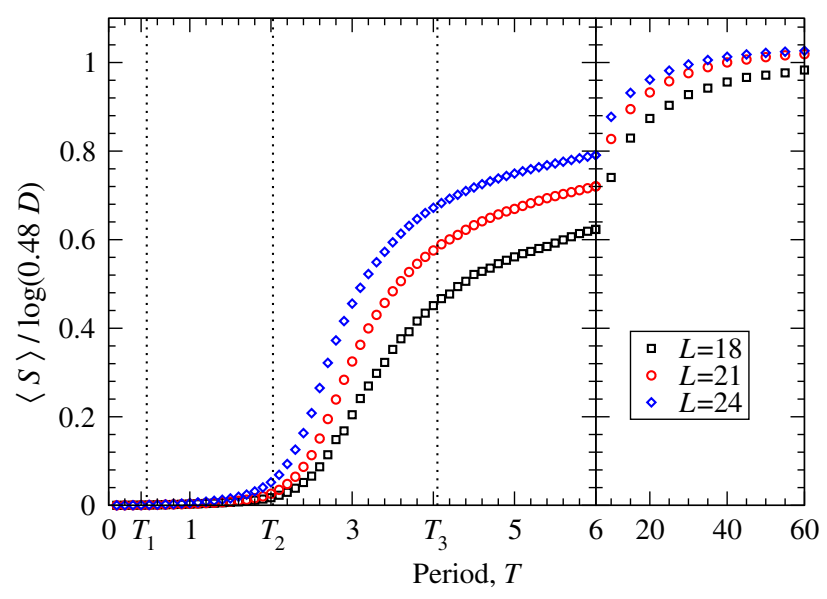

FIG. 4. Average entropy of the eigenstates $\langle S\rangle$ vs the period $T$. $\langle S\rangle / \ln (0.48 D)$ crosses over from zero to the COE prediction, which is reached earlier as $L$ increases. The variance $\left\langle\left(S_{n}-\langle S\rangle\right)^{2}\right\rangle^{1 / 2}$ is smaller than the size of the symbols used.

$\langle S\rangle$ grows monotonically with increasing $T$ and that the curves shift to the left (smaller periods) as the system size increases. The variance $\left\langle\left(S_{n}-\langle S\rangle\right)^{2}\right\rangle^{1 / 2}$ is smaller than the size of the symbols used in Fig. 4. This makes it apparent that, on average, different eigenstates $\left|\phi_{n}\right\rangle$ delocalize the same way in the basis $\left\{\left|m_{\text {ave }}\right\rangle\right\}$. Contrary to $\langle r\rangle$ in Fig. $2,\langle S\rangle$ in Fig. 4 is still changing for $T \geq T_{3}$. However, the COE prediction is reached for longer periods. This is not surprising since indicators based on the eigenvectors are known to suffer from stronger finite-size effects than level statistics indicators $[43,44]$.

\section{Heating towards infinite temperature}

The fact that $\hat{U}_{\text {cycle }}$ shares properties with matrices from the COE has important consequences for how the system adsorbs energy. To show it, we compute the expectation value of $\hat{H}_{\text {ave }}$ at long times $(t=N T$ for $N \rightarrow \infty)$. This can be done using the so-called diagonal ensemble $[41,42]$

$$
\begin{aligned}
\left\langle\hat{H}_{\text {ave }}\right\rangle(t=N T) & =\left\langle\psi_{0}\left|\left(\hat{U}_{\text {cycle }}^{\dagger}\right)^{N} \hat{H}_{\text {ave }}\left(\hat{U}_{\text {cycle }}\right)^{N}\right| \psi_{0}\right\rangle \\
& \approx \sum_{n}\left|\left\langle\psi_{0} \mid \phi_{n}\right\rangle\right|^{2}\left\langle\phi_{n}\left|\hat{H}_{\text {ave }}\right| \phi_{n}\right\rangle,
\end{aligned}
$$

where $\left|\psi_{0}\right\rangle$ is the initial state. If $\hat{U}_{\text {cycle }}$ is COE like, all its eigenstates are close to random vectors and the eigenstate expectation values of few-body observables (such as $\hat{H}_{\text {ave }}$ ) are almost $n$ independent. As a result, the evaluation of Eq. (17) gives the same result one would obtain in a system at infinite temperature, and this occurs independently of the initial state selected. Instead, if $\hat{H}_{F}=\hat{H}_{\text {ave }}$, the system does not absorb energy under driving.

In Fig. 5, we show the energy absorbed at long times $Q$ vs the period $T$. We have normalized $Q$ so that -1 corresponds to no energy absorption and 0 corresponds to the final energy being equal to that at infinite temperature:

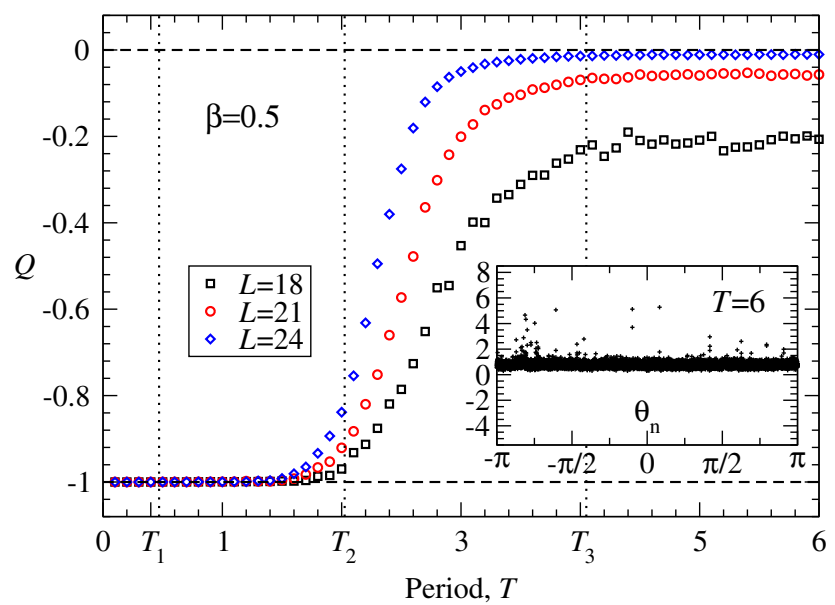

FIG. 5. Absorbed energy. (Main) Absorbed energy at long (infinite in our calculation) times $Q$ [see Eq. (18)] vs the driving period $T$. The initial state is a thermal state (with respect to $\hat{H}_{\text {ave }}$ ) with $\beta=0.5$. (Inset) Expectation values $\left\langle\phi_{n}\left|\hat{H}_{\text {ave }}\right| \phi_{n}\right\rangle$ vs the exact phases $\theta_{n}$ for $L=24$ and $T=6$.

$$
Q=\frac{\left\langle\hat{H}_{\mathrm{ave}}\right\rangle(t \rightarrow \infty)-\left\langle\hat{H}_{\mathrm{ave}}\right\rangle_{\beta=0}}{\left\langle\hat{H}_{\mathrm{ave}}\right\rangle_{\beta=0}-\left\langle\hat{H}_{\mathrm{ave}}\right\rangle(t=0)} .
$$

The initial state is taken to be in thermal equilibrium (with respect to $\hat{H}_{\text {ave }}$ ) with $\beta=0.5$. Namely, $\left|\psi_{0}\right\rangle\left\langle\psi_{0}\right|=$ $Z^{-1} \sum_{m} e^{-\beta E_{m}^{\text {ave }}}\left|m_{\text {ave }}\right\rangle\left\langle m_{\text {ave }}\right|$, where $Z$ is the partition function. Figure 5 shows that, with increasing $T, Q$ approaches zero as expected. In addition, the value of $T$ at which $Q$ saturates (close) to zero decreases with increasing system size.

As discussed above, the system reaching the equivalent of an infinite temperature state at long times can be traced back to the fact that, when $\hat{U}_{\text {cycle }}$ shares properties with matrices of the $\mathrm{COE}$, the expectation value of few-body observables is nearly independent of the specific Floquet eigenstate. This can be seen in the inset in Fig. 5, in which we plot the expectation values $\left\langle\phi_{n}\left|\hat{H}_{\text {ave }}\right| \phi_{n}\right\rangle$ vs $\theta_{n}$ for $L=24$ and $T=6$.

An understanding of how those eigenstate expectation values behave for different values of $T$ can be gained from Fig. 6. There, we report results for six different values of $T$. For short periods, the Floquet eigenstates $\left|\phi_{n}\right\rangle$ are closely related to the eigenstates of $\hat{H}_{\text {ave }}$, and the simple relation

$$
\theta_{n}=\frac{T}{\hbar}\left\langle\phi_{n}\left|\hat{H}_{\mathrm{ave}}\right| \phi_{n}\right\rangle
$$

holds [see Fig. 6(a)]. We note that the phases $\theta_{n}$ might or might not span the entire "Floquet Brillouin zone," i.e., $[-\pi, \pi)$, depending on the value of the period $T$. This can also be seen in Fig. 6(a). For intermediate periods, there is a crossover regime in which the relation between $\theta_{n}$ and $\left\langle\phi_{n}\left|\hat{H}_{\text {ave }}\right| \phi_{n}\right\rangle$ is progressively lost with increasing $T$ [see Fig. 6(b)]. For long periods $T$, the eigenstate expectation 


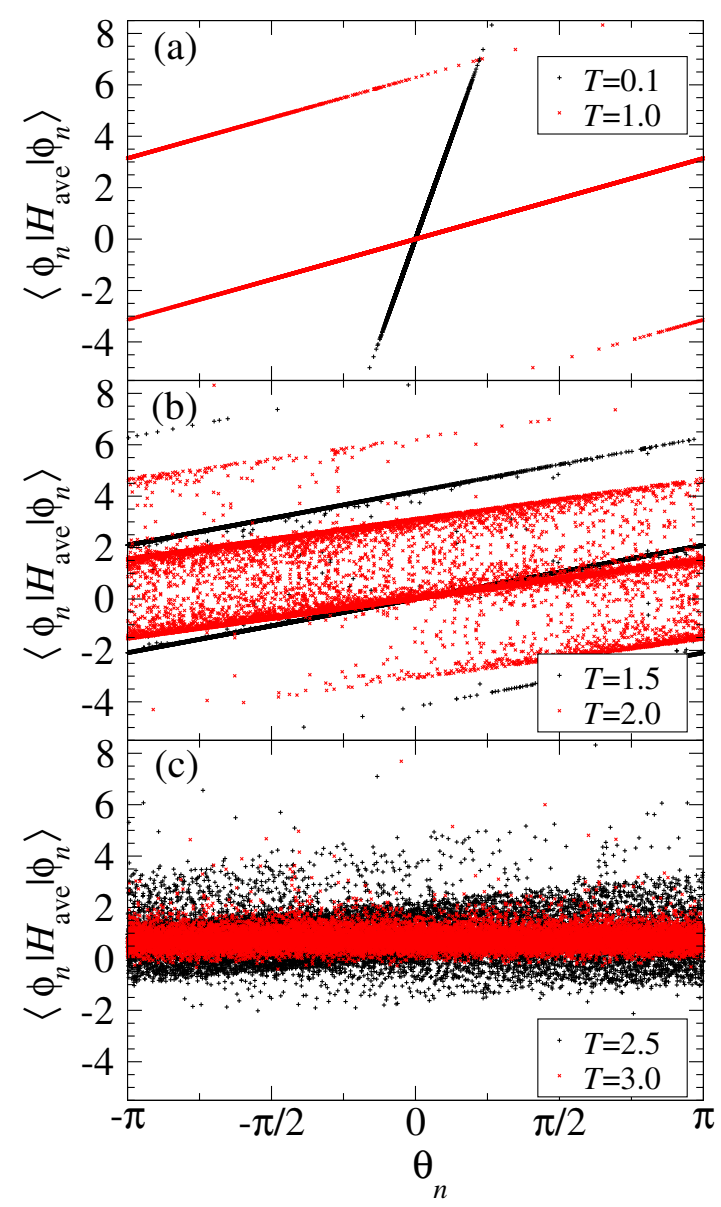

FIG. 6. Expectation values $\left\langle\phi_{n}\left|\hat{H}_{\text {ave }}\right| \phi_{n}\right\rangle$ vs the exact phases $\theta_{n}$ folded in $[-\pi, \pi)$ for $L=24$ and different driving periods $T$.

values $\left\langle\phi_{n}\left|\hat{H}_{\text {ave }}\right| \phi_{n}\right\rangle$ approach an $n$-independent value; i.e., they are not related to the values of the Floquet phases $\theta_{n}$ [see Fig. 6(c)]. Increasing the period beyond the ones reported in Fig. 6 further decreases the eigenstate-toeigenstate variation of $\left\langle\phi_{n}\left|\hat{H}_{\text {ave }}\right| \phi_{n}\right\rangle$, as one can realize by comparing the results in Fig. 6(c) with those in the inset in Fig. 5.

\section{DISCUSSION}

Our numerical results for finite systems indicate that $\hat{H}_{F}=\hat{H}_{\text {ave }}$ for small values of $T\left(T \lesssim T_{1}\right.$ in our study $)$, while $\hat{U}_{\text {cycle }}$ shares properties with matrices of the COE for large values of $T$ ( $T \gtrsim T_{3}$ in our study). In addition, there is a crossover regime (for intermediate periods $T_{1} \lesssim T \lesssim T_{3}$ ) in which some of the indicators used smoothly interpolate between the values obtained in the previous two regimes. In the intermediate regime, a drive may lead to states in which the average energy in a cycle fluctuates between consecutive cycles or becomes stationary but not equal to that of a system at infinite temperature. This could potentially be seen in current experiments with periodically driven ultracold atomic systems.
As we discussed in Sec. II, when the Magnus expansion converges, $\hat{H}_{F}$ is a local extensive Hamiltonian with fewbody interactions, which is incompatible with the phases of the eigenvalues of $\hat{U}_{\text {cycle }}$ exhibiting level repulsion for nonvanishing values of $T$ in thermodynamically large systems. Therefore, the fact that we see an onset of COE properties for $\hat{U}_{\text {cycle }}$ when $T \gtrsim T_{3} \propto 1 / \sqrt{L}$ indicates that the radius of convergence of the Magnus expansion is vanishingly small in thermodynamically large systems. As a result, for nonvanishingly small values of $T$, thermodynamically large systems reach the equivalent of an infinite temperature state at long driving times. Also, since the Floquet Hamiltonian $\hat{H}_{F}$ cannot be extensive with few-body interactions, one realizes that these systems offer a natural platform to investigate unique phenomena that may occur at intermediate times as a result of long-range many-body interactions in $\hat{H}_{F}$.

Remarkably, the Magnus expansion is guaranteed to converge only for $T \leq T_{1} \propto 1 / L$ [34,36], when the Floquet phases do not wrap around the "first Brillouin zone," i.e., $\theta_{n} \in[-\pi, \pi)$. Our results for $\langle r\rangle$, showing that $\langle r\rangle$ decreases approaching the POI prediction when $T$ increases for $T \lesssim$ $T_{2}$ (see Fig. 2), suggest instead that the radius of convergence of the Magnus expansion is $\sim T_{2} \propto 1 / \sqrt{L}$. This means that, for $T_{1}<T \lesssim T_{2}$, the Magnus expansion converges despite the fact that the Floquet phases wrap around the "first Brillouin zone." When the Magnus expansion converges, one can "unwrap" the Floquet phases $\theta_{n}$, which can be unambiguously defined in the "extended Brillouin zone," i.e., $\theta_{n} \in(-\infty, \infty)$ [see Fig. 6(a)], so that the relation $\theta_{n}=T \varepsilon_{n} / \hbar$ holds and both $\theta_{n}$ and the Floquet quasienergy $\varepsilon_{n}$ [see Eq. (3)] are extensive. When the Magnus expansion fails [20], it is not possible to unambiguously unwrap the Floquet phases, which are therefore only defined in the first Brillouin zone [see Fig. 6(c)]. Therefore, the breaking of the Magnus expansion coincides with the transition from a description in the extended Brillouin zone to one in the first Brillouin zone.

We expect that our results, in particular, the presence of the two limiting behaviors described above for short and long driving periods, will be valid beyond the specific model considered. In fact, at sufficiently short driving periods, the Magnus expansion is guaranteed to converge for any system with a bounded spectrum, and therefore $\hat{H}_{F} \approx \hat{H}_{\text {ave }}$. On the other hand, the same way that generic nonintegrable systems are expected to thermalize under unitary dynamics independently of the initial state selected [38-42], periodically driven generic nonintegrable systems are expected to heat up towards the equivalent of an infinite temperature state independently of the initial conditions. This expectation can be traced back to the second law of thermodynamics. Actually, if the driving period is longer than all relaxation time scales, then the system relaxes and effectively starts each driving cycle from a stationary state. It can be proven rigorously that, during any dynamical 
process that starts from a stationary state, the properly defined entropy can only increase [63]. Hence, at long times, the system reaches a state of maximum entropy, i.e., the equivalent of an infinite temperature state. This, in turn, requires $\hat{U}_{\text {cycle }}$ to have properties of the circular ensemble of a random matrix.

We note, however, that there are specific classes of models that escape this general expectation. In those systems, $\hat{H}_{F}$ is a well-behaved local Hamiltonian over a finite range of driving periods. Examples are as follows: (i) driven Hamiltonians that commute at different times, $\left[\hat{H}(\tau), \hat{H}\left(\tau^{\prime}\right)\right]=0$, which lead to $\hat{H}_{F}=\hat{H}_{\text {ave }}$ independently of $T$, (ii) fictitious time dependence that can be removed by a proper choice of the reference frame [12], and (iii) special cases in which multiple commutators of $\hat{H}(\tau)$ generate a finite algebra $[20,64]$. In the presence of strong quenched disorder, interacting quantum systems can become nonergodic. These systems, which are known as many-body localized (MBL), do not exhibit thermalization under unitary dynamics [57-62,65]. Currently, it is unknown whether MBL systems that are driven periodically in time by a global perturbation follow the general expectation discussed here (heat up indefinitely) or not [66]. The case of a MBL system driven periodically in time by local perturbations was studied in Ref. [26], where they were shown not to heat up indefinitely.

As discussed above, when the Magnus expansion does not converge, and $\hat{U}_{\text {cycle }}$ exhibits properties of random matrices of the circular ensemble, the system is expected to heat up towards the equivalent of an infinite temperature state at long times. We should stress, however, that the heating rate might be very small [31] and negligible on the time scale of a particular experiment. If this is the case, then the experiment could be described using the Magnus expansion truncated after the first few orders [67]. Moreover, at long times, the coupling between the system and the environment may become important and the system could approach a nonequilibrium steady state $[68,69]$ in which the energy absorbed from the driving is balanced by the energy dissipated into the environment [33]. Therefore, the observation of the equivalent of an infinite temperature state requires a separation of time scales [34]: $\tau_{\text {heating }} \ll \tau_{\text {bath }}$, where $\tau_{\text {heating }}^{-1}$ is the heating rate and $\tau_{\text {bath }}$ is the time scale at which the coupling to the bath becomes important.

\section{SUMMARY}

In summary, we have studied the long-time behavior of an isolated interacting spin chain that is periodically driven by sudden quenches. For finite systems, we found three possible regimes. For short driving periods, $\hat{H}_{F}$ converges to the time-averaged Hamiltonian $\hat{H}_{\text {ave }}$, while for long periods the evolution operator $\hat{U}_{\text {cycle }}$ has properties of matrices of the $\mathrm{COE}$ of random matrix theory. For intermediate periods, there is a crossover regime. We have provided evidence that, for thermodynamically large systems, the only regime that occurs for nonvanishing driving periods is the one in which $\hat{U}_{\text {cycle }}$ exhibits properties of random matrices of the COE. This results in the system heating up to the equivalent of an infinite temperature state at long times. Furthermore, we argued that in this regime, $\hat{H}_{F}$ cannot be an extensive Hamiltonian with few-body interactions.

Our findings for periodically driven systems are the equivalent of eigenstate thermalization for out-of-equilibrium many-body systems with time-independent Hamiltonians. While eigenstate thermalization ensures that thermalization occurs for generic interacting systems, the fact that the evolution operator in a periodically driven generic interacting system shares properties with matrices in the $\mathrm{COE}$ ensures that such systems thermalize to infinite temperature at long times.

\section{ACKNOWLEDGMENTS}

We thank M. Bukov, I. Iadecola, A. Polkovnikov, and L.F. Santos for stimulating discussions. This work was supported by the Office of Naval Research and by the Army Research Office.

Note added.-After posting our preprint on arXiv.org, preprints by Lazarides et al. [25] and Ponte et al. [26] also reported that periodically driven ergodic systems heat up, leading to the equivalent of an infinite-temperature state, in agreement with our results.

\section{APPENDIX A: DERIVATION OF EQ. (9)}

For completeness, we report the derivation of Eq. (9). Our presentation closely follows Ref. [31]. By plugging the ansatz (7) into the Schrödinger equation (6), one obtains

$$
\partial_{\tau} \exp \left[T \frac{\hat{H}_{\mathrm{eff}}(\tau)}{i \hbar}\right]=T \frac{\hat{H}(\tau)}{i \hbar} \exp \left[T \frac{\hat{H}_{\mathrm{eff}}(\tau)}{i \hbar}\right] .
$$

Using the mathematical identity

$$
\begin{aligned}
\partial_{\tau} \exp [\hat{B}(\tau)]= & \int_{0}^{1} d \alpha(\exp [\alpha \hat{B}(\tau)] \\
& \left.\times \partial_{\tau} \hat{B}(\tau) \exp [(1-\alpha) \hat{B}(\tau)]\right)
\end{aligned}
$$

to compute the derivative of an exponential operator [note that this expression is required because $\hat{H}_{\text {eff }}(\tau)$ at different times may not commute] results in

$$
\begin{aligned}
& \int_{0}^{1} d \alpha\left(\exp \left[\alpha T \frac{\hat{H}_{\mathrm{eff}}(\tau)}{i \hbar}\right] \partial_{\tau} \hat{H}_{\mathrm{eff}}(\tau) \exp \left[(1-\alpha) T \frac{\hat{H}_{\mathrm{eff}}(\tau)}{i \hbar}\right]\right) \\
& =\hat{H}(\tau) \exp \left[T \frac{\hat{H}_{\mathrm{eff}}(\tau)}{i \hbar}\right] .
\end{aligned}
$$


Multiplying both sides in the equation above by $\exp \left[-T\left(\hat{H}_{\mathrm{eff}}(\tau) / i \hbar\right)\right]$ from the right, one gets

$$
\begin{array}{r}
\int_{0}^{1} d \alpha\left(\exp \left[\alpha T \frac{\hat{H}_{\mathrm{eff}}(\tau)}{i \hbar}\right] \partial_{\tau} \hat{H}_{\mathrm{eff}}(\tau)\right. \\
\left.\quad \times \exp \left[-\alpha T \frac{\hat{H}_{\mathrm{eff}}(\tau)}{i \hbar}\right]\right)=\hat{H}(\tau) .
\end{array}
$$

We now close this equation between the exact (timedependent) eigenstates of $H_{\mathrm{eff}}(\tau)$,

$$
\hat{H}_{\mathrm{eff}}(\tau)\left|\phi_{n}(\tau)\right\rangle=\varepsilon_{n}(\tau)\left|\phi_{n}(\tau)\right\rangle
$$

and perform the integration over $\alpha$ to obtain

$$
\begin{aligned}
& \frac{i \hbar}{T} \frac{e^{\frac{T}{i \hbar}\left(\varepsilon_{n}(\tau)-\varepsilon_{m}(\tau)\right)}-1}{\varepsilon_{n}(\tau)-\varepsilon_{m}(\tau)}\left\langle\phi_{n}(\tau)\left|\partial_{\tau} \hat{H}_{\mathrm{eff}}(\tau)\right| \phi_{m}(\tau)\right\rangle \\
& \quad=\left\langle\phi_{n}(\tau)|\hat{H}(\tau)| \phi_{m}(\tau)\right\rangle
\end{aligned}
$$

To obtain Eq. (9), we substitute in Eq. (A3) the generalized Hellmann-Feynman theorem

$$
\begin{aligned}
& \left\langle\phi_{n}(\tau)\left|\partial_{\tau} \hat{H}_{\mathrm{eff}}(\tau)\right| \phi_{m}(\tau)\right\rangle \\
& \quad=\left(\varepsilon_{m}(\tau)-\varepsilon_{n}(\tau)\right)\left\langle\phi_{n}(\tau)\left|\partial_{\tau}\right| \phi_{m}(\tau)\right\rangle .
\end{aligned}
$$

We note that, while Eq. (A3) depends on the convention used in the definition of the Floquet quasienergy in Eq. (A2), the final equation (9) can be written exclusively in terms of the phase factors $e^{-i(T / \hbar) \epsilon_{n}(\tau)}=e^{-i \theta_{n}(\tau)}$, which are independent of the convention used in the definition of $\varepsilon_{n}(\tau)$.

\section{APPENDIX B: PHASE REPULSION IN CIRCULAR ENSEMBLES}

Here, we derive the probability distribution $P(r)$ in different ensembles. In Gaussian ensembles (GEs), $r$ is the ratio of consecutive energy gaps [see Eq. (12)], while in the CEs, $r$ is the ratio of consecutive phase gaps [see Eq. (15)]. The distribution $P(r)$ has been shown to be a sensitive and practical probe [52-54] of the integrability-tochaos transition [4]. For the GEs, the distribution $P(r)$ has recently been calculated [54] starting from the joint distribution of the eigenvalues of a $3 \times 3$ random matrix. We do the calculation for a $3 \times 3$ random matrix in the CEs.

The joint distribution for the phases in the circular orthogonal ensemble (COE), the circular unitary ensemble (CUE), and the circular symplectic ensemble (CSE) are [45-50]

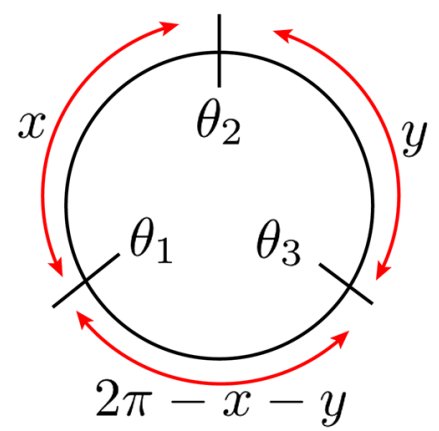

FIG. 7. Transformation between $\theta_{1}, \theta_{2}, \theta_{3}$ and $x, \theta_{2}, z$.

$$
\begin{aligned}
\rho\left(\theta_{1}, \cdots, \theta_{n}\right) & =\frac{1}{Z_{n, \beta}} \prod_{1 \leq k<j \leq n} \mid e^{i \theta_{k}}-e^{i \theta_{j} \mid \beta} \\
Z_{n, \beta} & =(2 \pi)^{n} \frac{\Gamma\left(\frac{\beta n}{2}+1\right)}{\left[\Gamma\left(\frac{\beta}{2}+1\right)\right]^{n}},
\end{aligned}
$$

and $\beta=1,2,4$ for COE, CUE, and CSE, respectively. For a $3 \times 3$ matrix, the expression simplifies to

$$
\begin{aligned}
\rho\left(\theta_{1}, \theta_{2}, \theta_{3}\right)= & \frac{2^{3 \beta}}{Z_{3, \beta}}\left|\sin \left(\frac{\theta_{1}-\theta_{2}}{2}\right)\right|^{\beta} \\
& \times\left|\sin \left(\frac{\theta_{1}-\theta_{3}}{2}\right)\right|^{\beta}\left|\sin \left(\frac{\theta_{2}-\theta_{3}}{2}\right)\right|^{\beta},
\end{aligned}
$$

where $\theta_{i} \in(0,2 \pi)$ [this follows from the normalization $\left.\iiint_{0}^{2 \pi} d \theta_{1} d \theta_{2} d \theta_{3} \rho\left(\theta_{1}, \theta_{2}, \theta_{3}\right)=1\right]$. We choose one of the six possible orderings of the angles, $\theta_{1} \leq \theta_{2} \leq \theta_{3}$, define the variable of interest in one of the three possible ways $\delta\left(\tilde{r}-\left(\theta_{2}-\theta_{1}\right) /\left(\theta_{3}-\theta_{2}\right)\right)$, and perform the change of variables $x=\theta_{2}-\theta_{1}, \theta_{2}, y=\theta_{3}-\theta_{2}$ to obtain

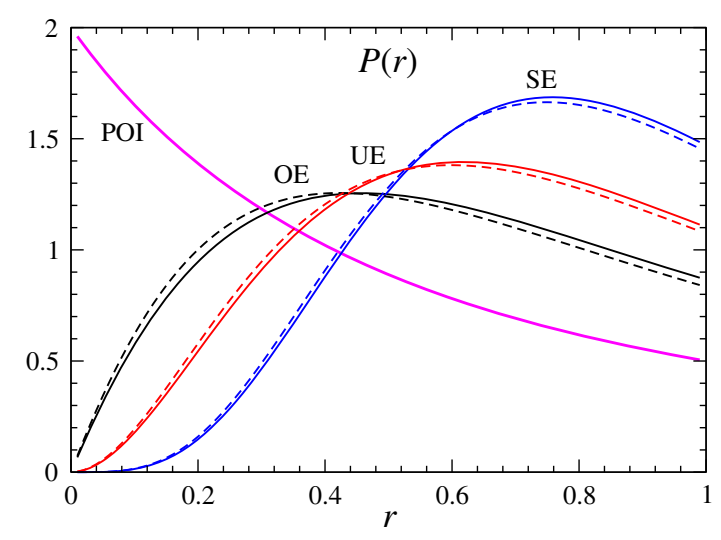

FIG. 8. The distribution $P(r)$ in the Gaussian and circular ensembles of random matrix theory and for a sequence of Poisson distributed random numbers. The label POI refers to Poisson, while the labels OE, UE, and SE refer to orthogonal, unitary, and symplectic ensembles, respectively. Continuous lines depict results for the Gaussian ensembles, while dotted lines depict results for the circular ensembles. 
TABLE I. Average value of $r$ in the Gaussian and circular ensembles of a random matrix. For comparison, the average value of $r$ for a sequence of Poisson distributed random numbers is $\langle r\rangle_{\mathrm{POI}}=0.386294$.

\begin{tabular}{lccc}
\hline \hline$\langle r\rangle$ & Orthogonal & Unitary & Symplectic \\
\hline Gaussian ensembles & 0.535898 & 0.602658 & 0.676168 \\
Circular ensembles & 0.526922 & 0.596543 & 0.671921 \\
\hline \hline
\end{tabular}

$$
\begin{aligned}
P(\tilde{r})= & \frac{2 \pi 6}{3} \frac{2^{3 \beta}}{Z_{3, \beta}} \int_{0}^{2 \pi} d x \int_{0}^{2 \pi-x} d y \delta\left(\tilde{r}-\frac{x}{y}\right) \\
& \times\left(\sin \frac{x}{2} \sin \frac{y}{2} \sin \frac{x+y}{2}\right)^{\beta},
\end{aligned}
$$

where the factor of $2 \pi$ comes from the integration $\int_{0}^{2 \pi} d \theta_{2}$, the factor of 6 comes from the choice of the ordering, and the factor of 3 comes from the choice of the observable. We also used the identity $\sin (2 \pi-x-y / 2)=\sin (x+y / 2)$. The limits of integration in Eq. (B1) come from the fact that $0 \leq x+y \leq 2 \pi$ (see Fig. 7).

In Eq. (B1), $\tilde{r} \in(0, \infty)$, and it is different from the $r$ introduced in Eqs. (12) and (15), which is defined in [0,1]. Luckily, the relation between $P(\tilde{r})$ and $P(r)$ is a simple one, $P(r)=2 P(\tilde{r}) \Theta(1-\tilde{r})$ [54]. Using Mathematica, we have performed the integration in Eq. (B1) exactly to obtain $P(r)$. For example, the distribution for the $\mathrm{COE}$ is

$$
\begin{aligned}
P_{\mathrm{COE}}(r)= & \frac{2}{3}\left(\frac{\sin \left(\frac{2 \pi r}{r+1}\right)}{2 \pi r^{2}}+\frac{1}{(r+1)^{2}}+\frac{\sin \left(\frac{2 \pi}{r+1}\right)}{2 \pi}\right. \\
& \left.-\frac{\cos \left(\frac{2 \pi}{r+1}\right)}{r+1}-\frac{\cos \left(\frac{2 \pi r}{r+1}\right)}{r(r+1)}\right) .
\end{aligned}
$$

For brevity, we do not present the expressions for the CUE and CSE.

In Fig. 8, we plot the distributions $P_{\mathrm{COE}}(r), P_{\mathrm{CUE}}(r)$, and $P_{\mathrm{CSE}}(r)$ and compare them with the corresponding distributions for the GEs $\left[P_{\mathrm{GOE}}(r), P_{\mathrm{GUE}}(r)\right.$, and $\left.P_{\mathrm{GSE}}(r)\right]$ and the distribution for a Poisson sequence of random numbers, $P_{\mathrm{POI}}(r)$. The distributions for the $\mathrm{CE}$ and the corresponding GE are very close for $3 \times 3$ matrices and are expected to coincide in the thermodynamic limit. In fact, the phases in the CEs are expected to have the same local fluctuation properties as the eigenvalues of the corresponding Gaussian ensembles (GEs) [50]. In Table I, we report the average value of $r$ for all the distributions considered.

[1] G. Casati, B. V. Chirikov, F. M. Izraelev, and Joseph Ford, Stochastic Behavior of a Quantum Pendulum under a Periodic Perturbation, in Stochastic Behavior in Classical and Quantum Hamiltonian Systems, Lecture Notes in Physics, Vol. 93, edited by Giulio Casati and Joseph Ford (Springer, Berlin Heidelberg, 1979), pp. 334-352.
[2] B. V. Chirikov, F. M. Izrailev, and D. L. Shepelyansky, Dynamical Stochasticity in Classical and Quantum Mechanics, Sov. Sci. Rev. Sect. C 2, 209 (1981).

[3] S. Fishman, D. R. Grempel, and R. E. Prange, Chaos, Quantum Recurrences, and Anderson Localization, Phys. Rev. Lett. 49, 509 (1982).

[4] L.E. Reichl, The Transition to Chaos: Conservative Classical Systems and Quantum Manifestations (Springer, New York, 2004).

[5] P. L. Kapitza, Dynamic Stability of a Pendulum When its Point of Suspension Vibrates, Sov. Phys. JETP 21, 588 (1951).

[6] H. W. Broer, I. Hoveijn, M. van Noort, C. Sim, and G. Vegter, The Parametrically Forced Pendulum: A Case Study in 1 1/2 Degree of Freedom, J. Dynamics Diff. Eqs. 16, 897 (2004).

[7] Z. Ovadyahu, Suppression of Inelastic Electron-Electron Scattering in Anderson Insulators, Phys. Rev. Lett. 108, 156602 (2012).

[8] S. Iwai, M. Ono, A. Maeda, H. Matsuzaki, H. Kishida, H. Okamoto, and Y. Tokura, Ultrafast Optical Switching to a Metallic State by Photoinduced Mott Transition in a Halogen Bridged Nickel-Chain Compound, Phys. Rev. Lett. 91, 057401 (2003).

[9] S. Kaiser, C. R. Hunt, D. Nicoletti, W. Hu, I. Gierz, H. Y. Liu, M. Le Tacon, T. Loew, D. Haug, B. Keimer, and A. Cavalleri, Optically Induced Coherent Transport Far above $T_{c}$ in Underdoped $\mathrm{YBa}_{2} \mathrm{Cu}_{3} \mathrm{O}_{6+\delta}$, Phys. Rev. B 89, 184516 (2014).

[10] T. Oka and H. Aoki, Photovoltaic Hall Effect in Graphene, Phys. Rev. B 79, 081406 (2009).

[11] T. Kitagawa, T. Oka, A. Brataas, L. Fu, and E. Demler, Transport Properties of Nonequilibrium Systems under the Application of Light: Photoinduced Quantum Hall Insulators without Landau Levels, Phys. Rev. B 84, 235108 (2011).

[12] T. Iadecola, D. Campbell, C. Chamon, C.-Y. Hou, R. Jackiw, S.-Y. Pi, and S. V. Kusminskiy, Materials Design from Nonequilibrium Steady States: Driven Graphene as a Tunable Semiconductor with Topological Properties, Phys. Rev. Lett. 110, 176603 (2013).

[13] N.H. Lindner, G. Refael, and V. Galitski, Floquet Topological Insulator in Semiconductor Quantum Wells, Nat. Phys. 7, 490 (2011).

[14] Y. H. Wang, H. Steinberg, P. Jarillo-Herrero, and N. Gedik, Observation of Floquet-Bloch States on the Surface of a Topological Insulator, Science 342, 453 (2013).

[15] T. Prosen, Time Evolution of a Quantum Many-Body System: Transition from Integrability to Ergodicity in the Thermodynamic Limit, Phys. Rev. Lett. 80, 1808 (1998).

[16] T. Prosen, Quantum Invariants of Motion in a Generic Many-Body System, J. Phys. A 31, L645 (1998).

[17] T. Prosen, Ergodic Properties of a Generic Nonintegrable Quantum Many-Body System in the Thermodynamic Limit, Phys. Rev. E 60, 3949 (1999).

[18] T. Prosen, General Relation between Quantum Ergodicity and Fidelity of Quantum Dynamics, Phys. Rev. E 65, 036208 (2002).

[19] M. Mierzejewski and P. Prelovšek, Nonlinear Current Response of an Isolated System of Interacting Fermions, Phys. Rev. Lett. 105, 186405 (2010). 
[20] L. DAlessio and A. Polkovnikov, Many-Body Energy Localization Transition in Periodically Driven Systems, Ann. Phys. (Amsterdam) 333, 19 (2013).

[21] S. De Sarkar, R. Sensarma, and K. Sengupta, A Perturbative Renormalization Group Approach to Driven Quantum Systems, J. Phys. Condens. Matter 26, 325602 (2014).

[22] C. A. Parra-Murillo, J. Madroñero, and S. Wimberger, Two-Band Bose-Hubbard Model for Many-Body Resonant Tunneling in the Wannier-Stark System, Phys. Rev. A 88, 032119 (2013).

[23] C. A. Parra-Murillo and S. Wimberger, Manifold Approach for a Many-Body Wannier-Stark System: Localization and Chaos in Energy Space, Acta Phys. Pol. A 124, 1091 (2013).

[24] K. Ji and B. V. Fine, Nonthermal Statistics in Isolated Quantum Spin Clusters after a Series of Perturbations, Phys. Rev. Lett. 107, 050401 (2011).

[25] A. Lazarides, A. Das, and R. Moessner, Equilibrium States of Generic Quantum Systems Subject to Periodic Driving, Phys. Rev. E 90, 012110 (2014).

[26] P. Ponte, A. Chandran, Z. Papic, and D. A. Abanin, Periodically Driven Ergodic and Many-Body Localized Quantum Systems, Ann. Phys. (Amsterdam) (2014), doi: 10.1016/j.aop.2014.11.008.

[27] S. Adachi, M. Toda, and K. Ikeda, Quantum-Classical Correspondence in Many-Dimensional Quantum Chaos, Phys. Rev. Lett. 61, 659 (1988).

[28] B. Gadway, J. Reeves, L. Krinner, and D. Schneble, Evidence for a Quantum-to-Classical Transition in a Pair of Coupled Quantum Rotors, Phys. Rev. Lett. 110, 190401 (2013).

[29] A. Eckardt, C. Weiss, and M. Holthaus, SuperfluidInsulator Transition in a Periodically Driven Optical Lattice, Phys. Rev. Lett. 95, 260404 (2005).

[30] S. Koghee, L.-K. Lim, M. O. Goerbig, and C. M. Smith, Merging and Alignment of Dirac Points in a Shaken Honeycomb Optical Lattice, Phys. Rev. A 85, 023637 (2012).

[31] M. M. Maricq, Application of Average Hamiltonian Theory to the NMR of Solids, Phys. Rev. B 25, 6622 (1982).

[32] E. B. Fel'dman, On the Convergence of the Magnus Expansion for Spin Systems in Periodic Magnetic Fields, Phys. Lett. A 104, 479 (1984).

[33] M. Langemeyer and M. Holthaus, Energy Flow in Periodic Thermodynamics, Phys. Rev. E 89, 012101 (2014).

[34] M. Bukov, L. D'Alessio, and A. Polkovnikov, Universal High-Frequency Behavior of Periodically Driven Systems: From Dynamical Stabilization to Floquet Engineering, arXiv:1407.4803.

[35] W. Magnus, On the Exponential Solution of Differential Equations for a Linear Operator, Commun. Pure Appl. Math. 7, 649 (1954).

[36] S. Blanes, F. Casas, J. A. Oteo, and J. Ros, The Magnus Expansion and Some of Its Applications, Phys. Rep. 470, 151 (2009).

[37] C. J. Joachain, Quantum Collision Theory (North-Holland Pub. Co., Amsterdam and Elsevier Science, New York, 1975).

[38] M. Srednicki, Chaos and Quantum Thermalization, Phys. Rev. E 50, 888 (1994).
[39] J. M. Deutsch, Quantum Statistical Mechanics in a Closed System, Phys. Rev. A 43, 2046 (1991).

[40] M. Srednicki, The Approach to Thermal Equilibrium in Quantized Chaotic Systems, J. Phys. A 32, 1163 (1999).

[41] M. Rigol, V. Dunjko, and M. Olshanii, Thermalization and Its Mechanism for Generic Isolated Quantum Systems, Nature (London) 452, 854 (2008).

[42] E. Khatami, G. Pupillo, M. Srednicki, and M. Rigol, Fluctuation-Dissipation Theorem in an Isolated System of Quantum Dipolar Bosons after a Quench, Phys. Rev. Lett. 111, 050403 (2013).

[43] L. F. Santos and M. Rigol, Onset of Quantum Chaos in OneDimensional Bosonic and Fermionic Systems and Its Relation to Thermalization, Phys. Rev. E 81, 036206 (2010).

[44] L. F. Santos and M. Rigol, Localization and the Effects of Symmetries in the Thermalization Properties of OneDimensional Quantum Systems, Phys. Rev. E 82, 031130 (2010).

[45] F. J. Dyson, Statistical Theory of the Energy Levels of Complex Systems. I, J. Math. Phys. (N.Y.) 3, 140 (1962).

[46] F. J. Dyson, Statistical Theory of the Energy Levels of Complex Systems. II, J. Math. Phys. (N.Y.) 3, 157 (1962).

[47] F. J. Dyson, Statistical Theory of the Energy Levels of Complex Systems. III, J. Math. Phys. (N.Y.) 3, 166 (1962).

[48] M. L. Mehta, Random Matrices, Vol. 142, 3rd ed. (Elsevier, Amsterdam, The Netherlands, 2004).

[49] F. Haake, Quantum Signatures of Chaos, 3rd ed. (Springer, Berlin, 2010).

[50] T. Guhr, A. Mller-Groeling, and H. A. Weidenmller, Random-Matrix Theories in Quantum Physics: Common Concepts, Phys. Rep. 299, 189 (1998).

[51] D. Li, Y. Dong, R. G. Ramos, J. D. Murray, K. MacLean, A. E. Dementyev, and S. E. Barrett, Intrinsic Origin of Spin Echoes in Dipolar Solids Generated by Strong $\pi$ Pulses, Phys. Rev. B 77, 214306 (2008).

[52] V. Oganesyan and D. A. Huse, Localization of Interacting Fermions at High Temperature, Phys. Rev. B 75, 155111 (2007).

[53] G. Biroli, A. C. Ribeiro-Teixeira, and M. Tarzia, Difference between Level Statistics, Ergodicity and Localization Transitions on the Bethe Lattice, arXiv:1211.7334.

[54] Y. Y. Atas, E. Bogomolny, O. Giraud, and G. Roux, Distribution of the Ratio of Consecutive Level Spacings in Random Matrix Ensembles, Phys. Rev. Lett. 110, 084101 (2013).

[55] T. A. Brody, J. Flores, J. B. French, P. A. Mello, A. Pandey, and S.S. M. Wong, Random-Matrix Physics: Spectrum and Strength Fluctuations, Rev. Mod. Phys. 53, 385 (1981).

[56] J. M. G. Gómez, R. A. Molina, A. Relaño, and J. Retamosa, Misleading Signatures of Quantum Chaos, Phys. Rev. E 66, 036209 (2002).

[57] D. M. Basko, I. L. Aleiner, and B. L. Altshuler, Metalinsulator Transition in a Weakly Interacting Many-Electron System with Localized Single-Particle States, Ann. Phys. (Amsterdam) 321, 1126 (2006).

[58] B. L. Altshuler, Y. Gefen, A. Kamenev, and L. S. Levitov, Quasiparticle Lifetime in a Finite System: A Nonperturbative Approach, Phys. Rev. Lett. 78, 2803 (1997). 
[59] I. V. Gornyi, A. D. Mirlin, and D. G. Polyakov, Interacting Electrons in Disordered Wires: Anderson Localization and Low-t Transport, Phys. Rev. Lett. 95, 206603 (2005).

[60] V. Oganesyan and D. A. Huse, Localization of Interacting Fermions at High Temperature, Phys. Rev. B 75, 155111 (2007).

[61] M. Žnidarič, T. ž Prosen, and P. Prelovšek, Many-Body Localization in the Heisenberg xxz. Magnet in a Random Field, Phys. Rev. B 77, 064426 (2008).

[62] A. Pal and D. A. Huse, Many-Body Localization Phase Transition, Phys. Rev. B 82, 174411 (2010).

[63] A. Polkovnikov, Microscopic Diagonal Entropy and Its Connection to Basic Thermodynamic Relations, Ann. Phys. (Amsterdam) 326, 486 (2011).

[64] V. Galitski, Quantum-to-Classical Correspondence and Hubbard-Stratonovich Dynamical Systems: A Lie-Algebraic Approach, Phys. Rev. A 84, 012118 (2011).
[65] E. Khatami, M. Rigol, A. Relaño, and A. M. Garcia-Garcia, Quantum Quenches in Disordered Systems: Approach to Thermal Equilibrium without a Typical Relaxation Time, Phys. Rev. E 85, 050102(R) (2012).

[66] S. Iyer, V. Oganesyan, G. Refael, and D. A. Huse, ManyBody Localization in a Quasiperiodic System, Phys. Rev. B 87, 134202 (2013).

[67] G. S. Boutis, P. Cappellaro, H. Cho, C. Ramanathan, and D. G. Cory, Pulse Error Compensating Symmetric Magic-Echo Trains, J. Magn. Reson. 161, 132 (2003).

[68] H. Dehghani, T. Oka, and A. Mitra, Dissipative Floquet Topological Systems, Phys. Rev. B 90, 195429 (2014).

[69] Z. Gu, H. A. Fertig, D. P. Arovas, and A. Auerbach, Floquet Spectrum and Transport through an Irradiated Graphene Ribbon, Phys. Rev. Lett. 107, 216601 (2011). 